\title{
Dynamical Behavior of a 3D Jerk System with a Generalized Memristive Device
}

\author{
Wei Feng $\mathbb{D}^{1,2}$ Yi-Gang He $\mathbb{D},{ }^{1}$ Chun-Lai Li $\mathbb{D}^{,},{ }^{3}$ Xun-Min Su, ${ }^{3}$ and Xiao-Qing Chen ${ }^{3}$ \\ ${ }^{1}$ School of Electrical Engineering and Automation, Hefei University of Technology, Hefei 230009, China \\ ${ }^{2}$ School of Mathematics and Computer Science, Panzhihua University, Panzhihua 617000, China \\ ${ }^{3}$ College of Physics and Electronics, Hunan Institute of Science and Technology, Yueyang 414006, China
}

Correspondence should be addressed to Yi-Gang He; yghe@hfut.edu.cn and Chun-Lai Li; hnistlichl@163.com

Received 18 May 2018; Accepted 28 June 2018; Published 19 August 2018

Academic Editor: Viet-Thanh Pham

Copyright (C) 2018 Wei Feng et al. This is an open access article distributed under the Creative Commons Attribution License, which permits unrestricted use, distribution, and reproduction in any medium, provided the original work is properly cited.

As a new type of electronic components, a memristive device is receiving worldwide attention and can enrich the dynamical behaviors of the oscillating system. In this paper, we propose a 3D jerk system by introducing a generalized memristive device. It is found that the dynamical behaviors of the system are sensitive to the initial conditions even the system parameters are fixed, which results in the coexistence of multiple attractors. And there exists different transition behaviors depending on the selection of the parameters and initial values. Thereby, it is one important type of the candidate system for secure communication since the reconstruction of accurate state space becomes more difficult. Moreover, we build a hardware circuit and the experimental results effectively confirm the theoretical analyses.

\section{Introduction}

As the fourth basic circuit element besides resistance, inductance, and capacitance, the memristor was postulated by Chua in 1971, to link the charge with the flux [1]. And the concept of the memristive system was further extended by Chua and Kang [2]. However, research on the memristor had not been received much interest until 2008, when the solid-state realization of the memristor was reported by the scholars in Hewlett-Packard Laboratory [3]. The memristor belongs to a nonvolatile two-terminal passive device with variable quantity called memristance, which connects the electric flux applied to the device with the electric charge passing through in a particular direction. When the electrical field in the memristor is removed, the memristance may remain unchanged, thus holding memory characteristic $[4,5]$. It was proved that the memory characteristic of the memristor could not be reproduced by any combination of the other three fundamental elements. Therefore, the memristor could be in fact considered as a new electronic element in electrical circuit theory, which is generally called as the fourth element [1].
In 2009, Chua put forward the other two hypothetical circuit elements called memcapacitor and meminductor from the nanoworld [6-8], which have closely the same characteristics as the memristor depending on the past states through which the system has evolved, so they are all called to be memory circuit elements.

As the new circuit element, the memristor has potential applications in the construction of the new generation of computers and memories and has been found to have significant applications in memristor oscillators, memristorbased neural networks, and memristor-based charge pump locked loops [9, 10]. Additionally, a memristive circuit is propitious to generate chaotic signal for the intrinsic nonlinearity and plasticity properties [11-13]. Different from the conventional nonlinear systems, the most significant feature of the memristor-based nonlinear system is that the long-term dynamical behaviors extremely rely on the initial state of the memristor, which leads to the emergence of multistability or coexisting many attractors $[14,15]$. The phenomenon of multistability has attracted a lot of research enthusiasm recently. In many cases, the multistability exists in dynamical systems with stable equilibrium, no-equilibrium, 


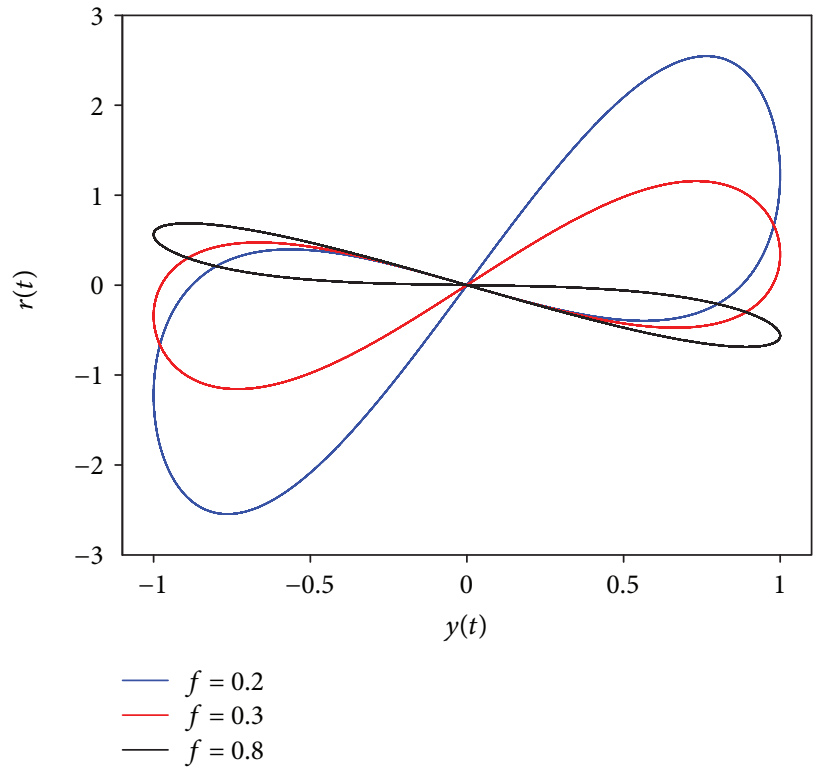

(a)

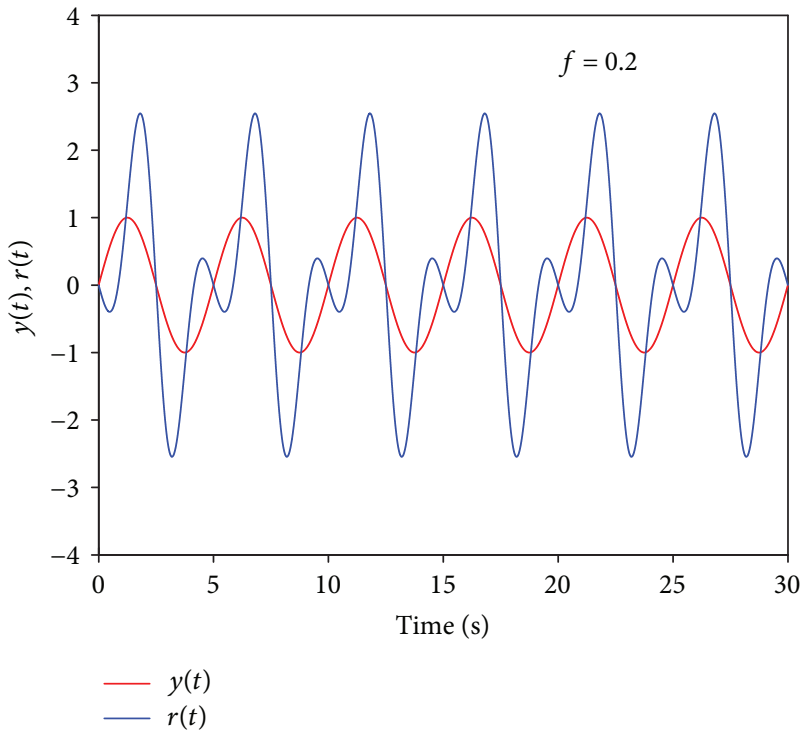

(b)

Figure 1: (a) Relationship curves of input and output under different frequencies and (b) the time-domain waveforms when $f=0.2 \mathrm{~Hz}$.

or a line of equilibrium, in which one cannot use the Shilnikov method to explain the chaos [14-18].

In this paper, we introduce a generalized memristive device by extending the definition of the memristor and propose a $3 \mathrm{D}$ jerk system based on the memristive element. Basic dynamical properties of the jerk system are displayed and studied. Specially, the coexistence of multiple attractors is investigated by analyzing the bifurcation map, spectrum of Lyapunov exponent, and distribution of the stable region in the initial value space. And there exist different transition behaviors depending on the selection of the parameters and initial values. Thereby, this system exhibits a rich and complex dynamics relying on the system parameters, initial values, and time evolution, which are of significance for secure communication since the reconstruction of accurate state space becomes more difficult. Moreover, we build a hardware circuit and the experimental results effectively confirm the theoretical analysis.

\section{Generalized Memristive Device}

By extending the definition of the memristive system [2, 19-21], we introduce a generalized memristive device, depicted by the following relation:

$$
\begin{aligned}
\frac{d x}{d t} & =y, \\
r & =\left(x^{2}-2\right) y .
\end{aligned}
$$

In relation (1), $x(t)$ denotes the internal state variable of the memristive element; $y(t)$ and $r(t)$ are complementary constitutive variables representing the input and output, respectively.

To study the fingerprint, we consider a sinusoidal stimulus $y=h \sin (\omega t)=h \sin (2 \pi f t)$ with the amplitude $h$ and the frequency $\omega$ or $f$, connected across the terminals of the memristive element [22-24]. Thus, we have

$$
\begin{aligned}
x(t) & =\int_{-\infty}^{t} y(\tau) d \tau=\int_{-\infty}^{0} y(\tau) d \tau+\int_{0}^{t} y(\tau) d \tau \\
& =x(0)+\frac{h}{2 \pi f}[1-\cos (2 \pi f t)] .
\end{aligned}
$$

And we further obtain the output of the memristive element, as

$$
r=\left(x^{2}-2\right) y=\left[\left(x(0)+\frac{h}{2 \pi f}(1-\cos (2 \pi f t))\right)^{2}-2\right] h \sin (2 \pi f t) .
$$

As we know that the resulting output not only depends on the initial state of the memristive element but also depends on the frequency and amplitude of the sinusoidal input. Figure 1(a) depicts the relations of input and output of the memory element for sinusoidal stimulus at different frequencies, when $h=1$ and $x(0)=1$. Figure $1(\mathrm{~b})$ shows the corresponding time domain waveform of $f=0.2$. Figure 2(a) depicts the relations of input and output of the memory element with $h=1$ and $f=0.2$ under different initial states. Figure 2(b) depicts the relations of input and output of the memory element with $x(0)=1$ and $f=0.2$ under different amplitudes of the sinusoidal signal.

From the simulation results in Figures 1 and 2, it can be seen that the memory device is not passive and behaves as a linear negative commutator in the limit of infinite frequency. Besides, there exist at most two values of the output $r(t)$ for any designated input $y(t)[20]$. 


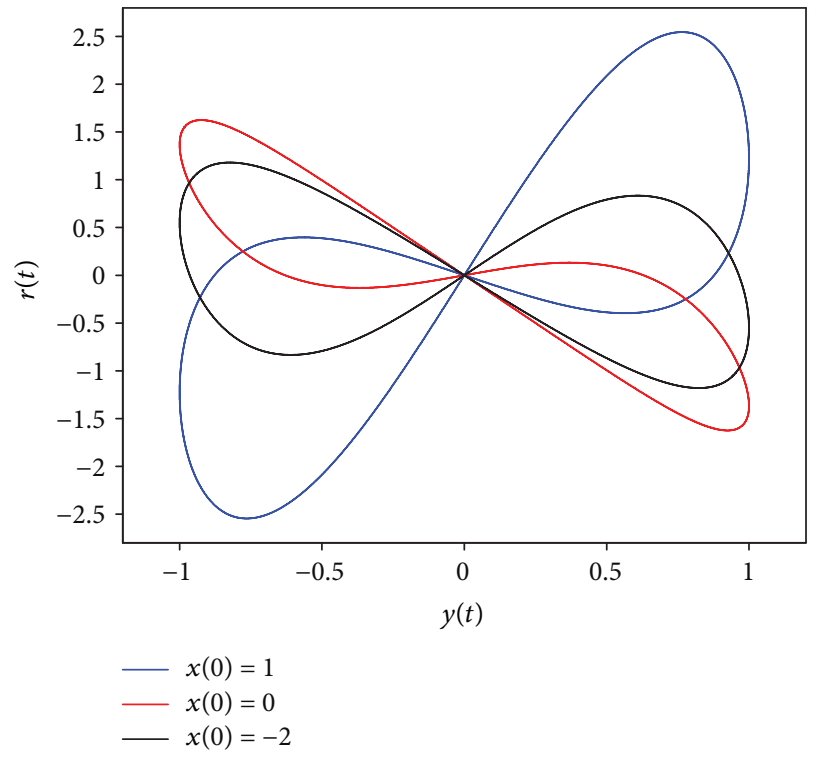

(a)

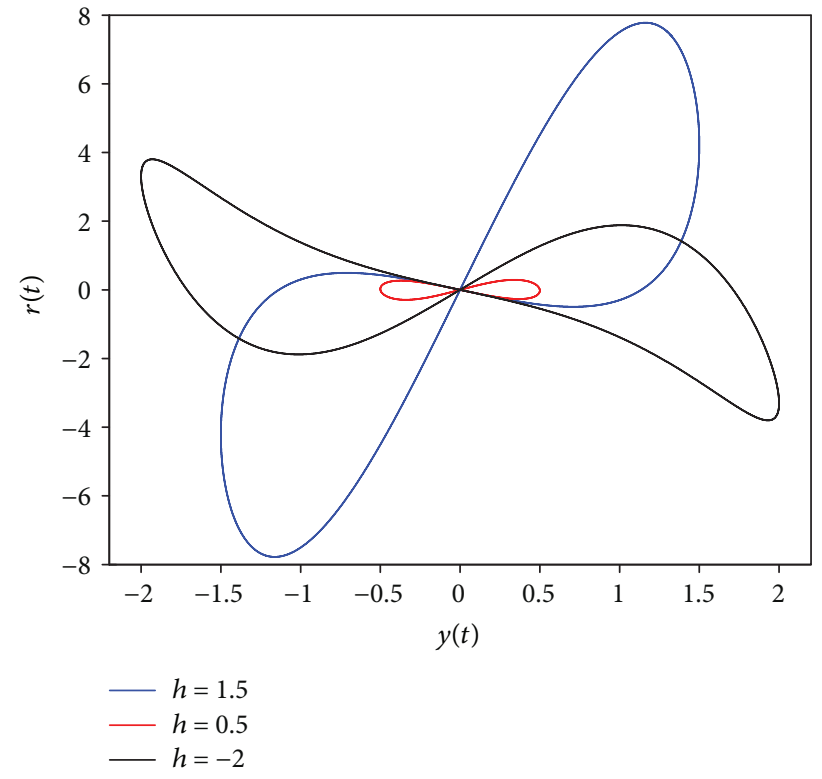

(b)

Figure 2: Relationship curves of input and output under (a) different initial states and (b) different amplitudes of the sinusoidal signal.

\section{Model of a Memristor-Based Jerk System}

Jerk system is of interest within the nonlinear dynamics domain for the simple mathematical form yet rich dynamics. A jerk system is depicted by the third-order ordinary differential equation as $d^{3} x / d t^{3}=J\left(d^{2} x / d t^{2}, d x / d t, x\right)$, in which the function $J(\cdot)$ is nonlinear. From the point of view of mechanics, the function $J(\cdot)$ corresponds to the first-time derivative of acceleration; thus, it is called to be a jerk or jolt [25-27].

In this work, we introduce a $3 \mathrm{D}$ jerk system which possesses the generalized memristive element of (1):

$$
\begin{aligned}
& \frac{d x}{d t}=y, \\
& \frac{d y}{d t}=d z, \\
& \frac{d z}{d t}=-a z+b x-c x^{3}+k r,
\end{aligned}
$$

where $r=\left(x^{2}-2\right) y, x, y, z$ are the state variables, and $a, b, c, k$ are the positive system parameters.

The volume contraction of system (4) can be described by the Lie derivative:

$$
\nabla V=\left(\frac{\partial \dot{x}}{\partial x}\right)+\left(\frac{\partial \dot{y}}{\partial y}\right)+\left(\frac{\partial \dot{z}}{\partial z}\right)=-a
$$

This signifies that the dissipativity of system (4) is negative. Thereby, the limit sets of system (4) will infinitely converge to zero volume, and the asymptotic motion at time through the flow will settle onto an attractor.

We obtain the three equilibrium points of system (4) as $E_{0}(0,0,0), E_{1}(\sqrt{b / c}, 0,0)$, and $E_{2}(-\sqrt{b / c}, 0,0)$, by simple mathematical derivation. When letting the parameter set
$P=(a, b, c, d, k)=(0.5,0.8,0.6,3.0,1)$, we get the typical equilibrium points and the corresponding characteristic roots, as below

$$
\begin{aligned}
E_{0}(0,0,0): & \lambda_{1}=-1.12415-1.88224 i, \\
\lambda_{2} & =-1.12415+1.88224 i, \lambda_{3}=1.2483, \\
E_{1,2}( \pm 1.1547,0,0): & \lambda_{1}=-2.54216, \lambda_{2}=0.771078-2.03122 i, \\
\lambda_{3} & =0.771078+2.03122 i .
\end{aligned}
$$

Therefore, the equilibrium point $E_{0}$ is a saddle node of index 1 , and the equilibrium points $E_{1}$ and $E_{2}$ are both saddle-focus points of index 2. Accordingly, the three equilibrium points are all instable.

With the parameter set $P$, we get the Lyapunov exponents as $0.165274,0$, and -0.662735 and the Kaplan-Yorke dimension as 2.2494, implying a fractional feature with chaotic behavior, as depicted in Figure 3.

\section{Dynamics of a Memristor-Based Jerk System}

4.1. Impact of System Parameters. We first select the parameter set $P$ except let $b$ vary in the region $(0.6,0.82)$, the bifurcation diagram and the Lyapunov exponent spectrum are displayed in Figure 4. It is known from Figure 4 that the system trajectory evolves from a fixed point to multiple period-doubling bifurcations, finally falls into a chaotic state. And there exists an obvious periodic window near $b=0.75$. The Feigenbaum's constant can be acquired by $F_{n}=\left(\rho_{n}-\rho_{n-1}\right) /\left(\rho_{n+1}-\rho_{n}\right)$, where $\rho_{n}$ is the critical parameter value at which a period-doubling bifurcation emerges. It is agreed that the ratio $F_{n}$ will converge to the ideal value 4.669 as $n$ increases. Thus, the ratio on the parameter $b$ is reckoned as $(0.7241-0.6782) /(0.7341-0.7241)=4.59$, which 


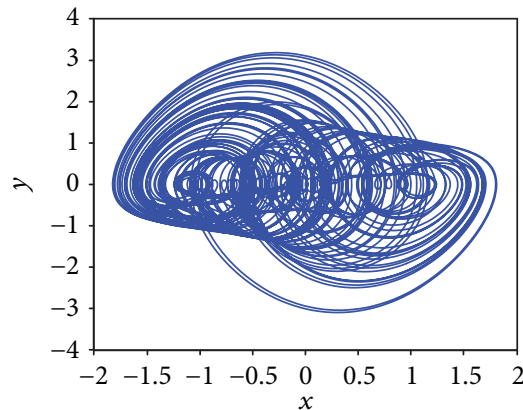

(a)

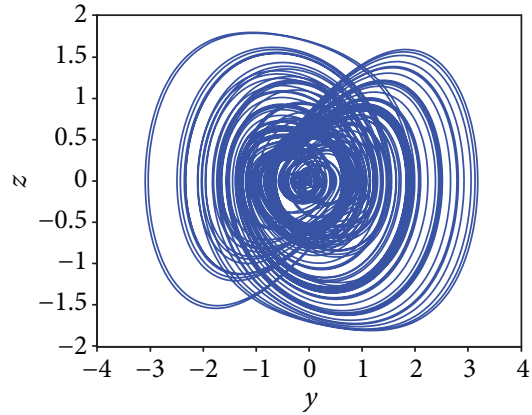

(b)

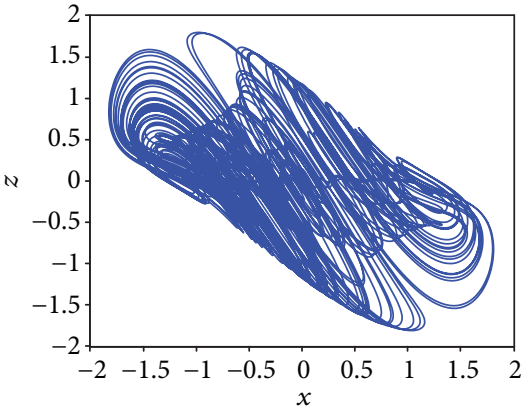

(c)

FIgURE 3: (a) $x-y$ phase portrait, (b) $y-x$ phase portrait, (c) $x-z$ phase portrait.

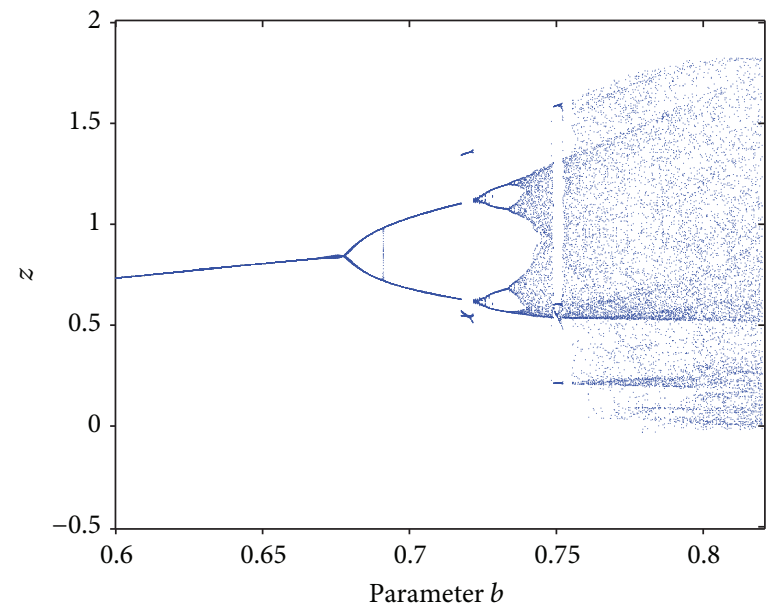

(a)

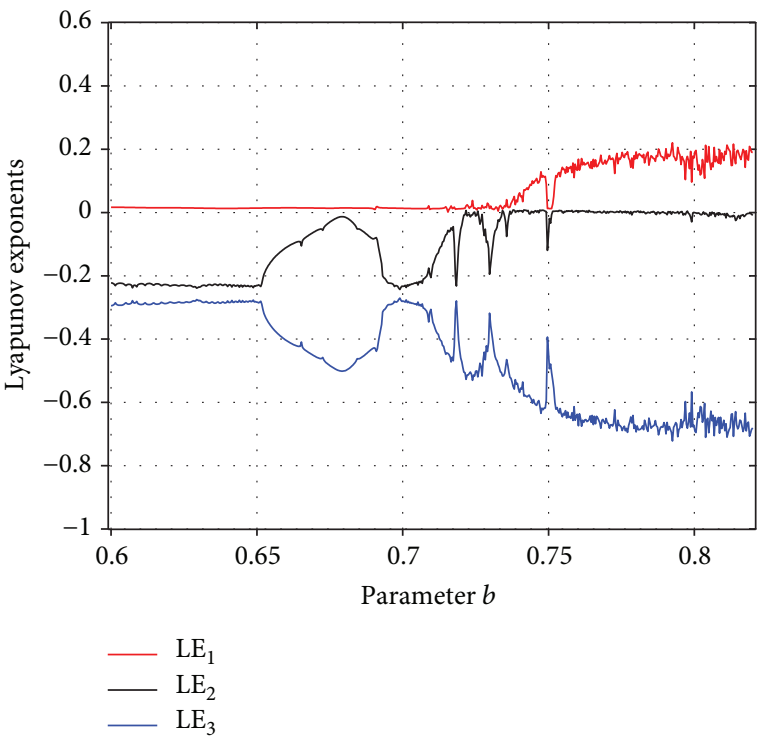

(b)

Figure 4: (a) Bifurcation diagram and (b) Lyapunov exponent spectrum versus parameter $b$.

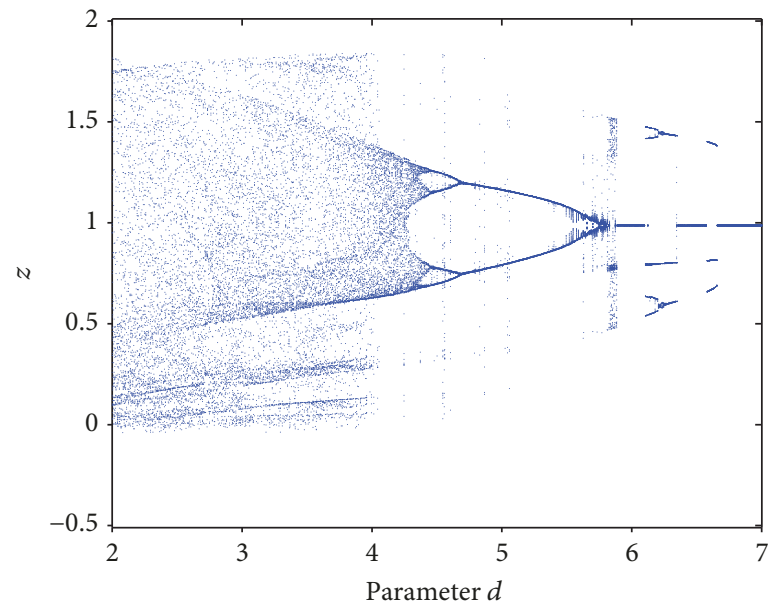

(a)

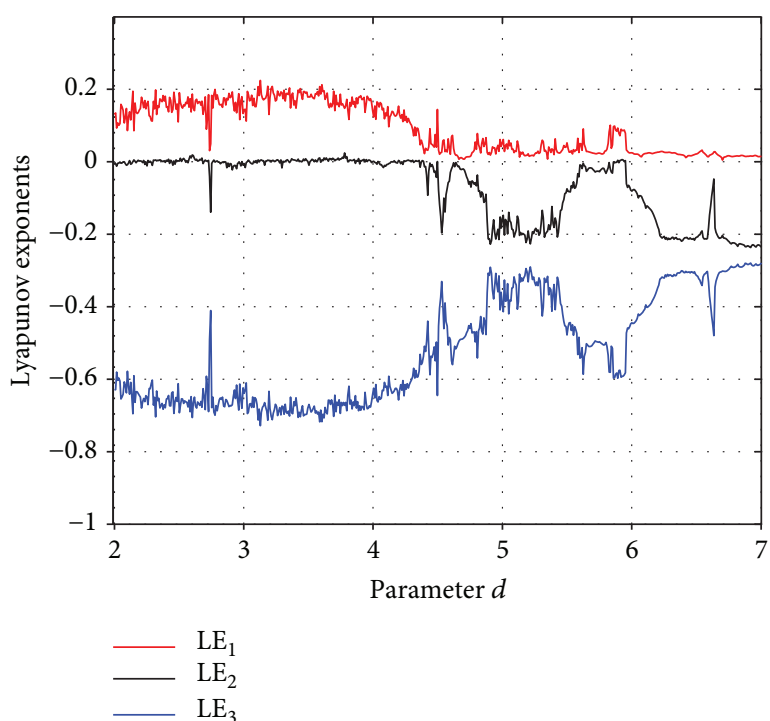

(b)

Figure 5: (a) Bifurcation diagram and (b) Lyapunov exponent spectrum versus parameter $d$. 


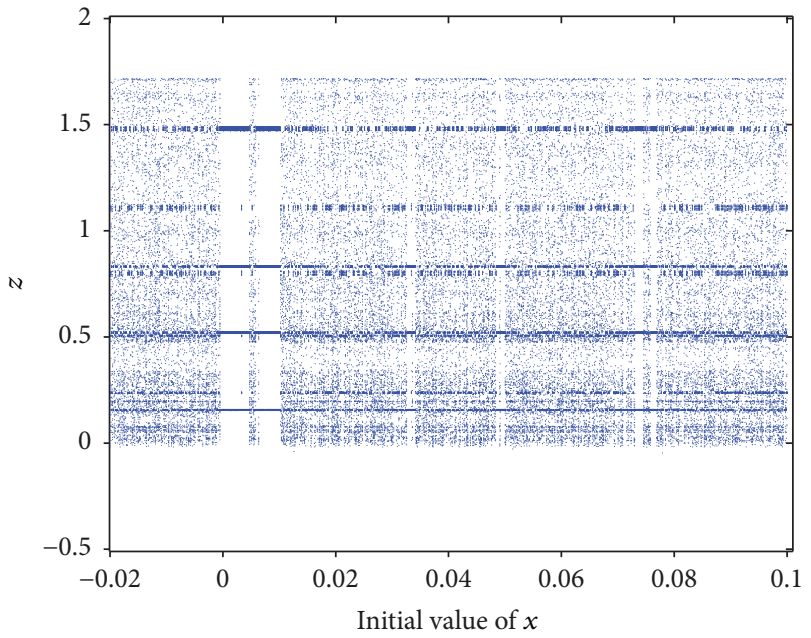

(a)

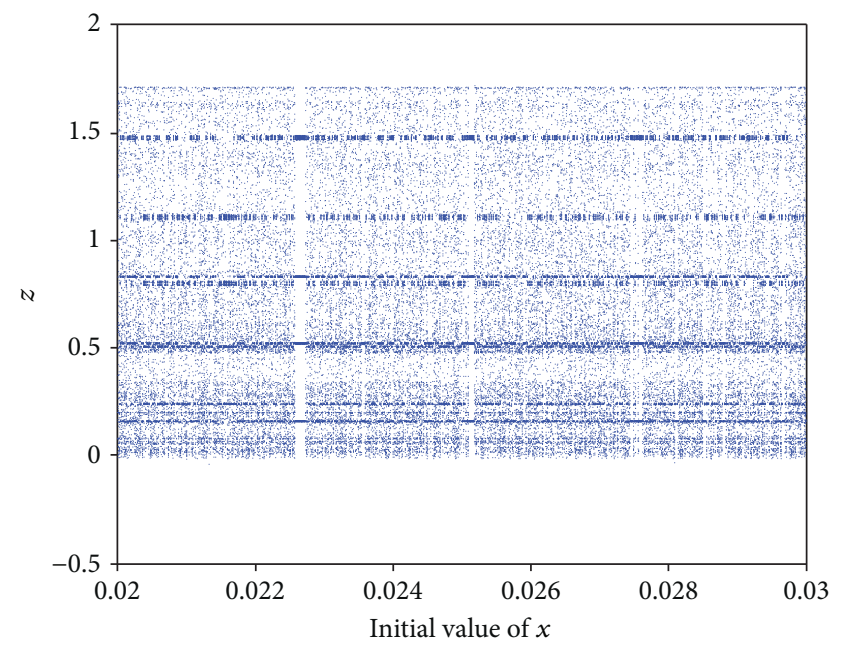

(b)

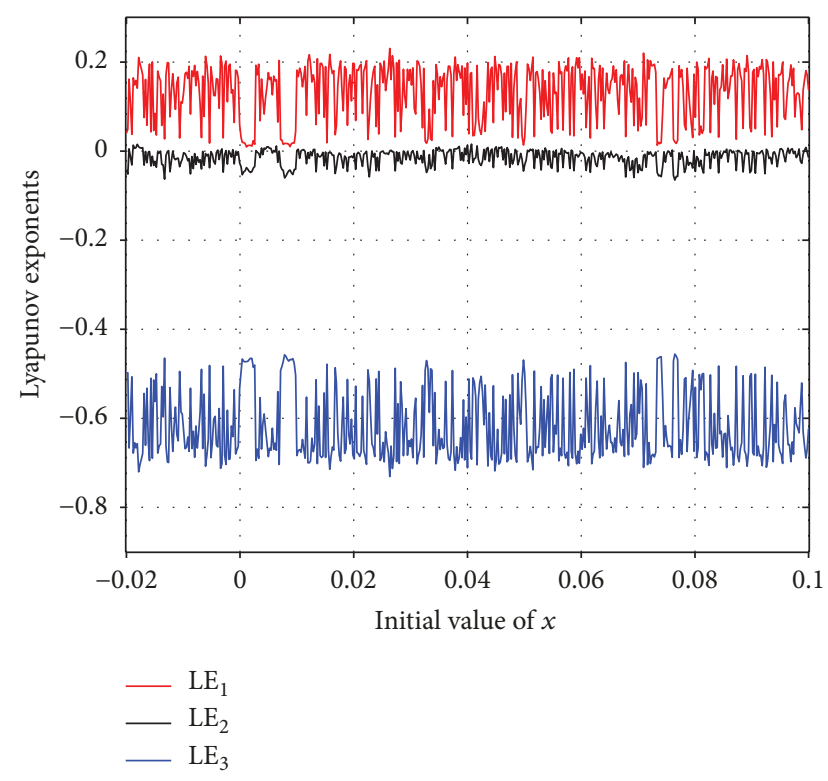

(c)

Figure 6: $(a, b)$ Bifurcation diagram and (c) Lyapunov exponent spectrum versus $x(0)$.

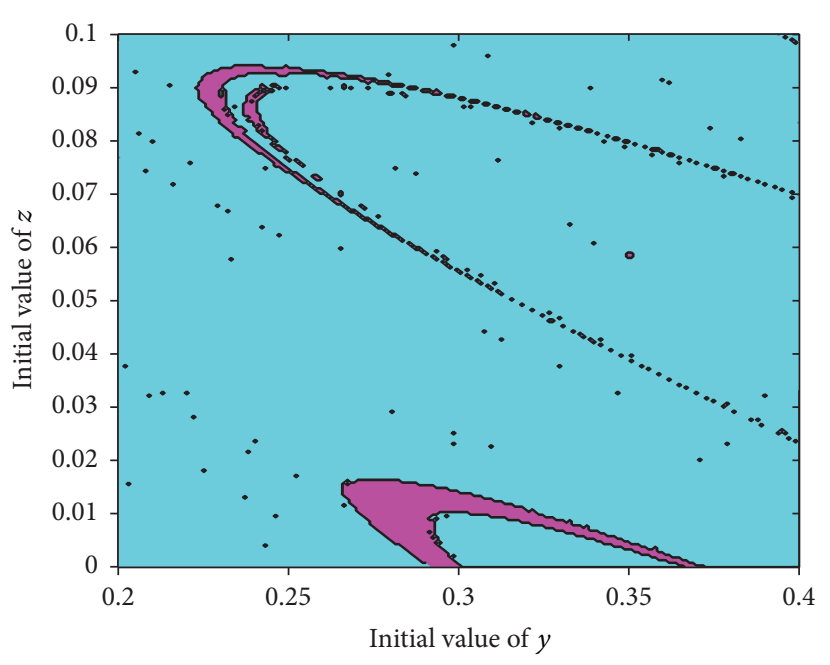

Figure 7: Dynamical map with $y(0)$ and $z(0)$.

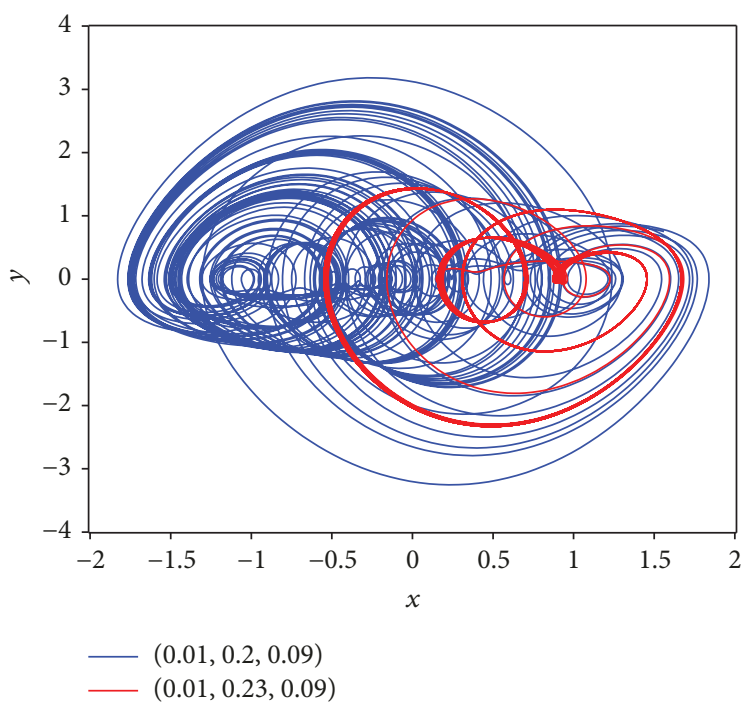

(a)

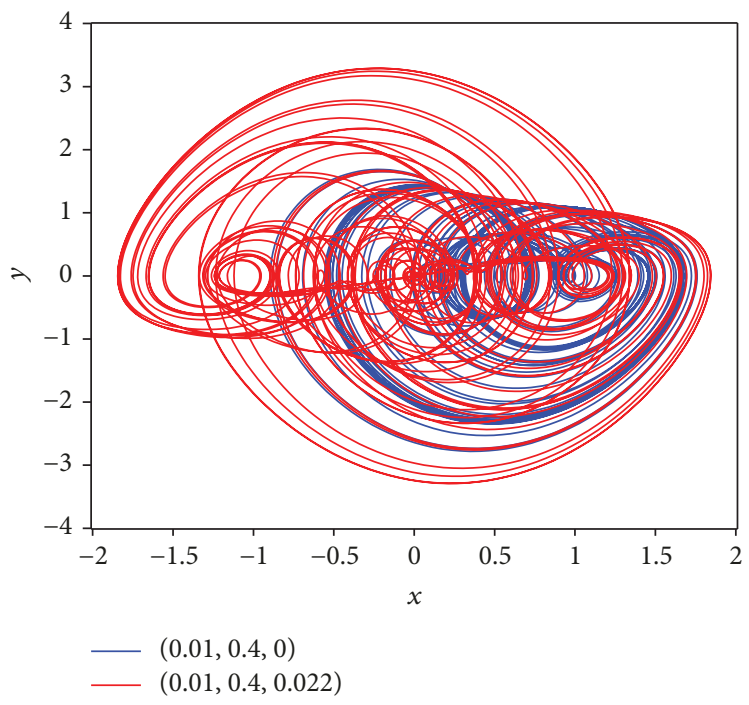

(b)

Figure 8: Attractors on $x-y$ plane with different initial conditions. 
TABLE 1: Multistable behavior of system (4) with $a=0.5, b=0.8, c=0.6, d=3$, and $k=0.95$.

\begin{tabular}{lcr}
\hline Initial condition & Lyapunov exponent & Dynamical behavior \\
\hline$(0.01,0.2,0.09)$ & $0.173334,0.009299,-0.682633$ & $2-S c r o l l$ chaotic attractor \\
$(0.01,0.23,0.09)$ & $0.0017705,-0.062944,-0.484761$ & Period-3 mode \\
$(0.01,0.4,0)$ & $0.050191,-0.0047652,-0.502539$ & 1 -Scroll chaotic attractor \\
$(0.01,0.4,0.022)$ & $0.148570,-0.001767,-0.646803$ & $2-S c r o l l$ chaotic attractor \\
\hline
\end{tabular}

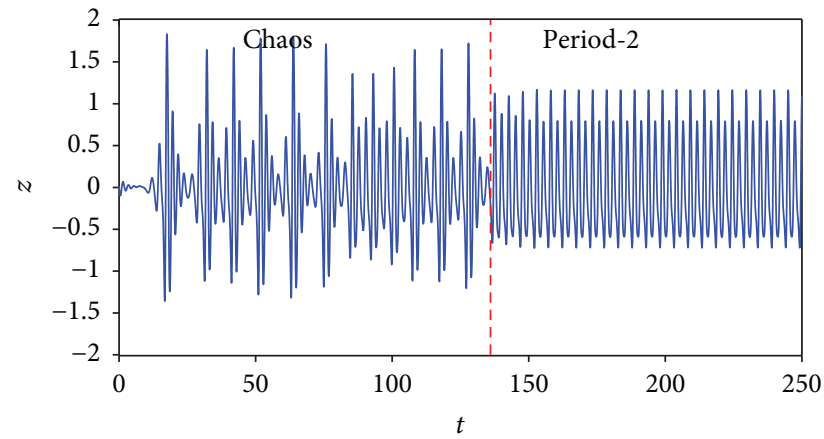

(a)

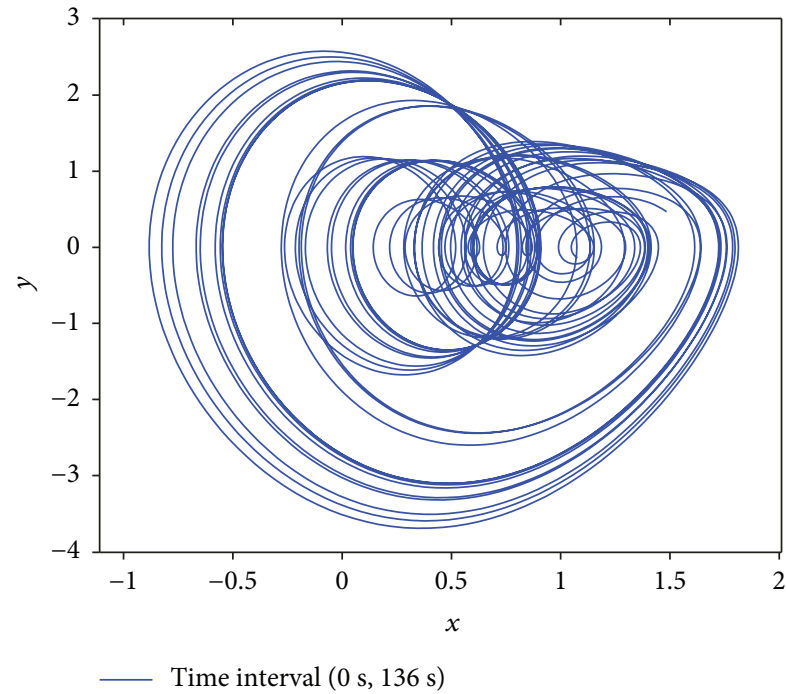

(b)

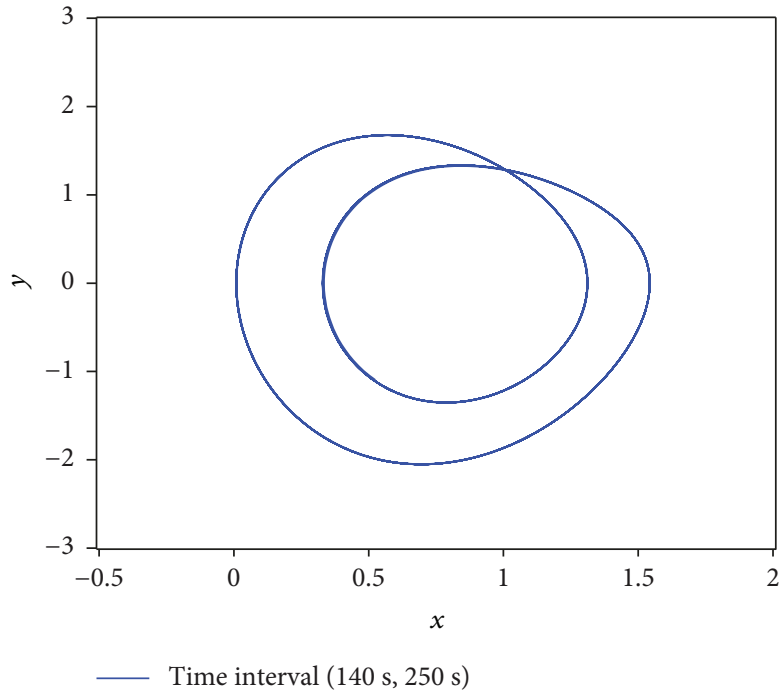

(c)

Figure 9: (a) Time-domain waveform of variable $z$ in the region of $(0 \mathrm{~s}, 250 \mathrm{~s})$; (b) the phase portrait in time interval of $(0 \mathrm{~s}, 136 \mathrm{~s})$; (c) the phase portrait in time interval of (140 s, $250 \mathrm{~s})$.

approximately equals to 4.669 with a relative deviation (decline) of $1.69 \%$.

Figure 5 depicts the bifurcation diagram and the Lyapunov exponent spectrum when selecting parameter set $P$ except $d$ varying in the region $[2,7]$. It is obvious that the dynamics of system (4) switch among the chaotic state and periodic orbit by inverse period-doubling Feigenbaum tree, with the increase of parameter $d$. And the Feigenbaum's constant is reckoned as $(5.732-4.666) /(4.666-4.445)=$ 4.8235 , which is approximately equal to 4.669 with a relative deviation (rise) of $3.31 \%$.

4.2. Coexisting Attractors. We assign the parameters of system (4) as $a=0.5, b=0.8, c=0.6, d=3$, and $k=0.95$ and take the initial conditions $y(0)=0.3$ and $z(0)=0.015$. When let $x(0)$ as the bifurcation parameter varying in the region $(-0.02,0.1)$, the bifurcation diagram and its Lyapunov exponent spectra are plotted in Figure 6. It is found that when initial value of $x$ increases from -0.02 , system (4) starts from a chaotic state and abruptly breaks into a periodic state via tangent bifurcation at -0.005 , and system turns into the normal chaotic state at 0.0035 then degrades into a periodic state at 0.0065 via chaos crisis; the periodic state continues until the tangent bifurcation at $x(0)=0.012$. As depicted by the enlarged view in Figure 6(b), similar switching process will happen in other ranges or smaller local ranges, showing a multifractal process.

When assigning the same parameters and $x(0)=0.01$, the dynamic dependence on both initial conditions $y(0)$ and $z(0)$ is also studied by the dynamical map, through 


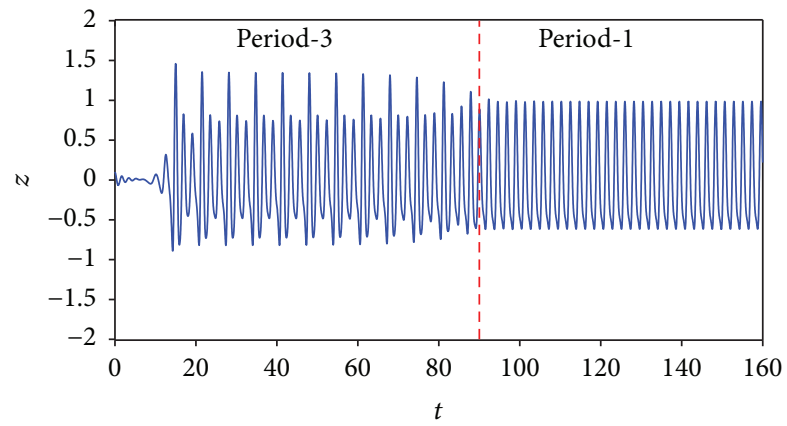

(a)

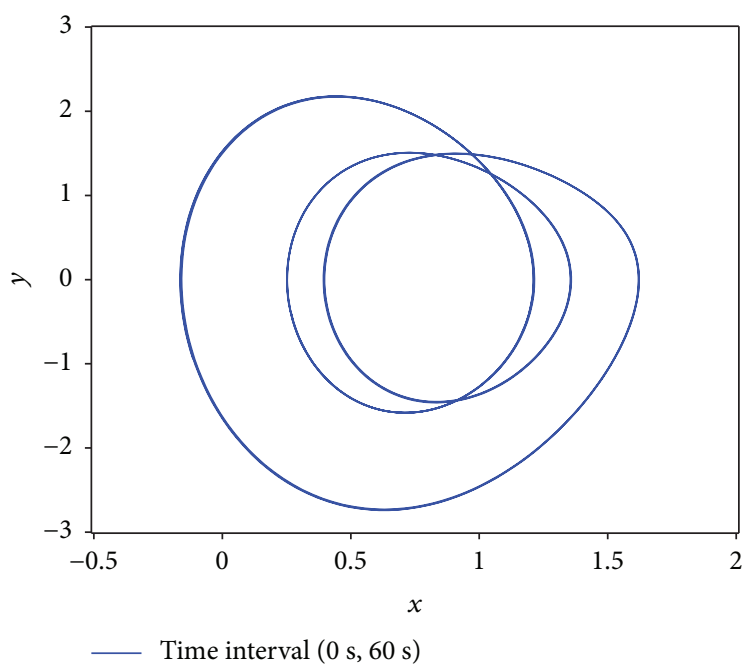

(b)

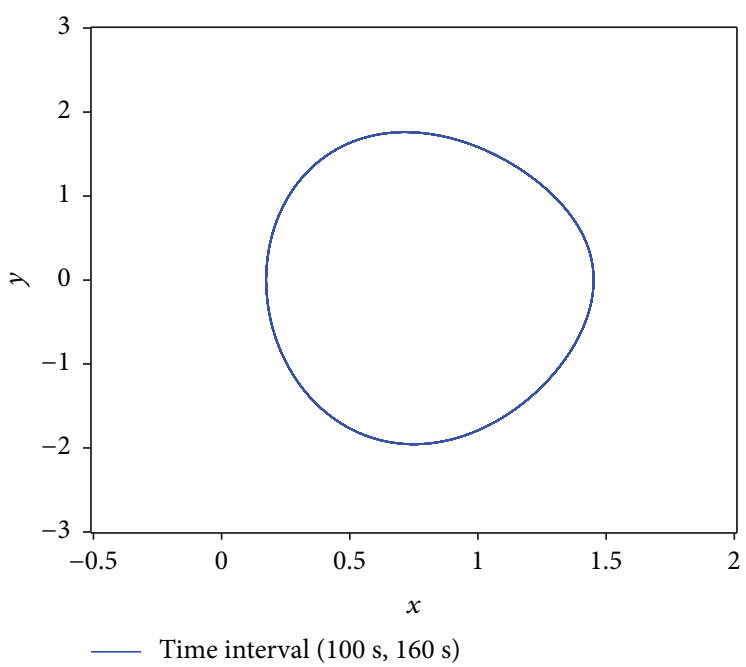

(c)

FIGURE 10: (a) Time-domain waveform of variable $z$ in the region of $(0 \mathrm{~s}, 160 \mathrm{~s})$; (b) the phase portrait in time interval of $(0 \mathrm{~s}, 60 \mathrm{~s})$; (c) the phase portrait in time interval of (100 s, $160 \mathrm{~s})$.

numerical simulation, as shown in Figure 7. In the dynamical map, the system is chaotic in the cyan region, stable in the pink region, and periodic in the blue region.

All these results of initial sensitivity indicate that there exists palpable feature of coexisting attractors in system (4). As the representative examples, Figure 8 displays the attractors on $x-y$ plane with $a=0.5, b=0.8, c=0.6, d=3$, and $k=0.95$ and different initial conditions. Figure 8(a) displays that the initial condition $(0.01,0.2,0.09)$ leads to a 2 -scroll chaotic mode and the initial condition $(0.01,0.23,0.09)$ leads to a period-3 mode. Figure $8(\mathrm{~b})$ displays that the initial condition $(0.01,0.4,0)$ leads to a 1 -scroll chaotic attractor and the initial condition $(0.01,0.4,0.022)$ leads to a 2 -scroll chaotic attractor. The typical multistable behaviors are summarized in Table 1.

4.3. Transient Dynamics. As discussed above, the dynamic mode of the reported system strongly depends not only on the parameters but also on the initial conditions. Another important phenomenon is that the dynamic mode also strongly depends on state evolution time, called to be the transition behavior.

Taking $a=0.5, b=0.8, c=0.6, d=5, k=1$ and the initial condition $(0.01,0.2,0.01)$, we depict the time trajectory in the region of $(0 \mathrm{~s}, 250 \mathrm{~s})$ and the phase portraits in time intervals of $(0 s, 136 s)$ and $(140 s, 250 s)$, as shown in Figure 9. As we find that the dynamics convert from transient chaos to period-2 behavior.

Taking $a=0.5, b=0.8, c=0.6, d=6.6, k=1$ and the initial condition $(0.01,0,0.1)$, we depict the time trajectory in the region of $(0 \mathrm{~s}, 160 \mathrm{~s})$ and the phase portraits in time intervals of $(0 s, 60 s)$ and $(100 s, 160 s)$, as shown in Figure 10, from which we find the dynamics transition from transient period-3 to period-1 behaviors.

Figure 11 depicts the case of $a=0.5, b=0.8, c=0.6, d=3$, $k=0.95$ and the initial condition $(0.01,0.26,0.01)$. As we find that the system (4) is chaotic in the region of $(0 \mathrm{~s}$, $1050 \mathrm{~s})$ and is in the mode of period- 4 in the region of (1050s, 2000s).

\section{Circuit Realization of a Memristor-Based Jerk System}

The circuit realization is important for the chaotic system, especially by adopting commercially common electronic components [28-30]. In this section, we build an electronic circuit to physically realize the reported system for different cases, based on the improved module-based technique 


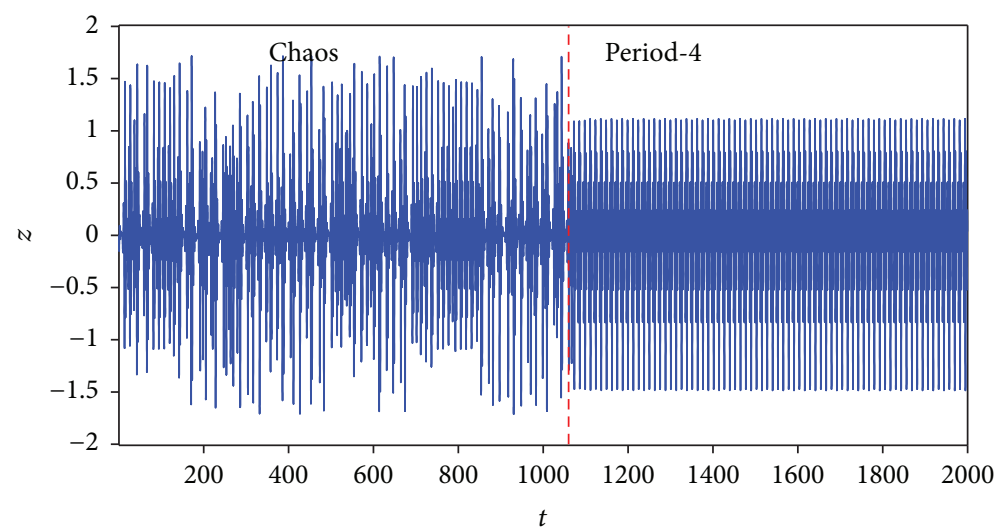

(a)

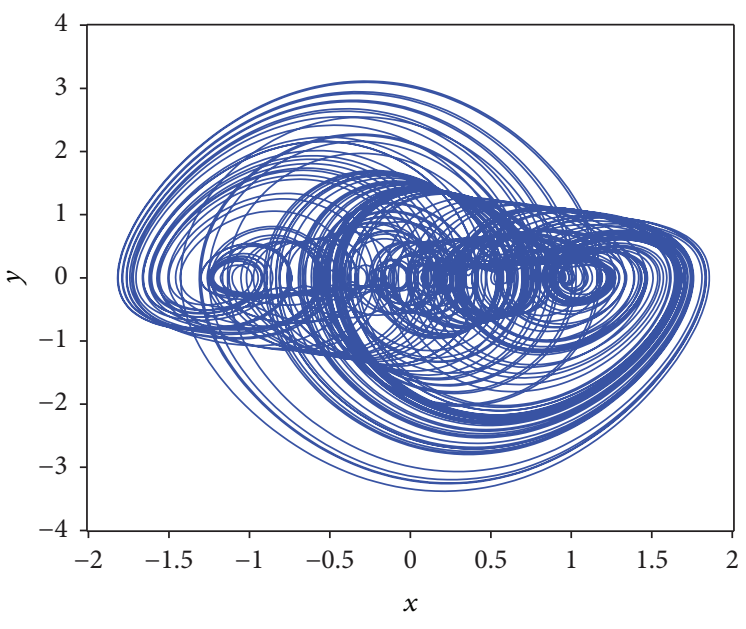

Time interval ( $0 \mathrm{~s}, 800 \mathrm{~s})$

(b)

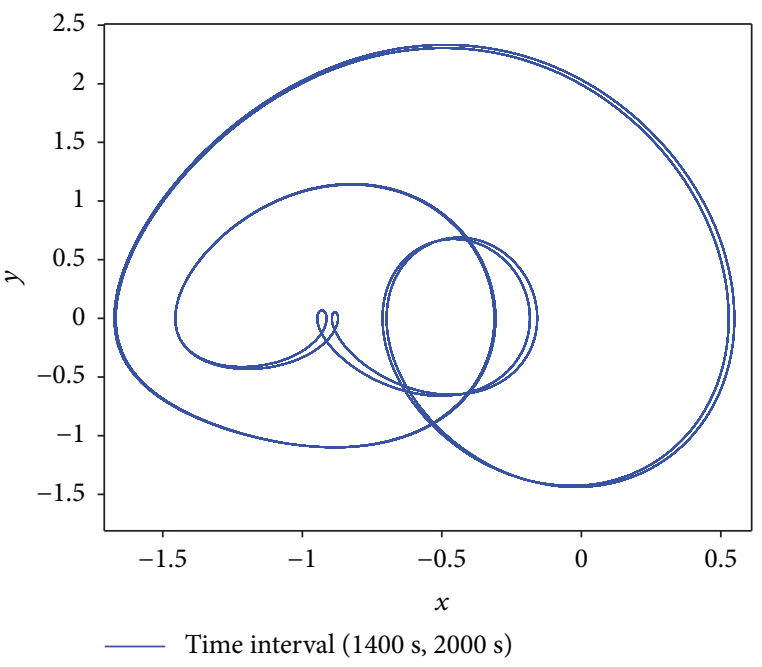

(c)

Figure 11: (a) Time-domain waveform of variable $z$ in the region of $(0 \mathrm{~s}, 2000 \mathrm{~s})$; (b) the phase portrait in time interval of $(0 \mathrm{~s}, 800 \mathrm{~s})$; (c) the phase portrait in time interval of (1400 s, $2000 \mathrm{~s})$.

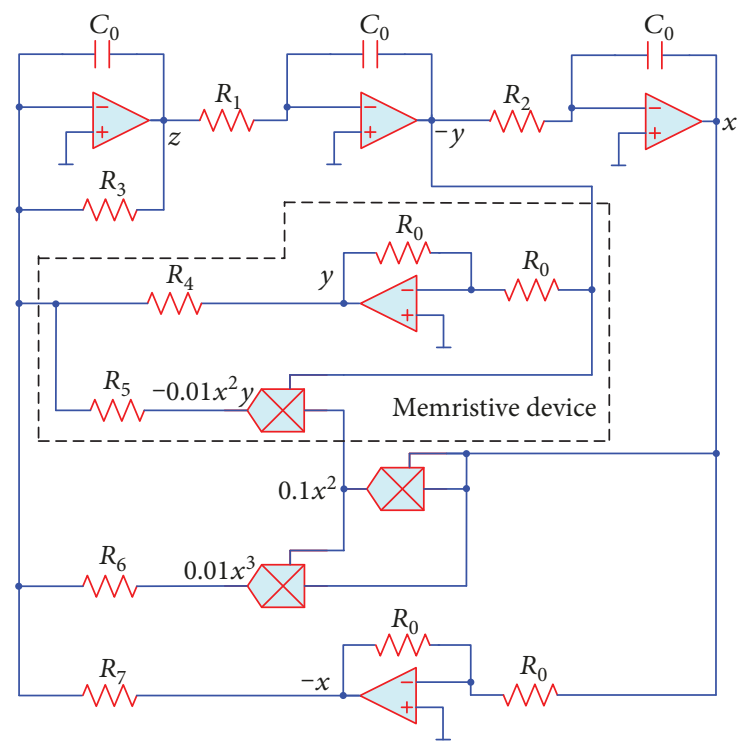

FIGURE 12: Electronic circuit realization of the emulating system (4).
[31-34]. The electronic circuit is designed as the jerk form by using the dimensionless state equations, as depicted in Figure 12, which is simple with less circuit elements. In this design, the operations of integral, addition, and inverse are realized by the operational amplifier TL082 chip and the multiply operation is realized by the AD633JN chip. Moreover, time-scale transformation is considered in our experiment to guarantee capturing of the wave effectively, which is determined by the time constant $R_{0} C_{0}$ in the integrator. Accordingly, we obtain the circuit state equation from Figure 12, as follows

$$
\begin{aligned}
\frac{d x}{d t}= & \frac{1}{R_{2} C_{0}} y, \\
\frac{d y}{d t}= & \frac{1}{R_{1} C_{0}} z, \\
\frac{d z}{d t}= & -\frac{1}{R_{3} C_{0}} z+\frac{1}{R_{7} C_{0}} x-\frac{1}{100 R_{6} C_{0}} x^{3} \\
& +\frac{1}{100 R_{5} C_{0}} x^{2} y-\frac{1}{R_{4} C_{0}} y .
\end{aligned}
$$




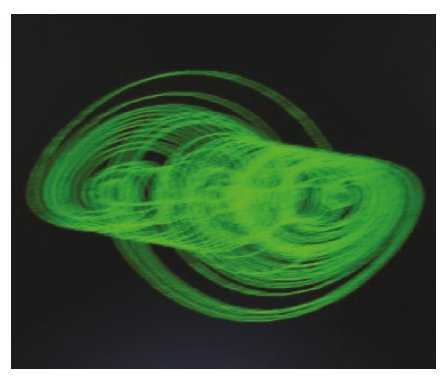

(a)

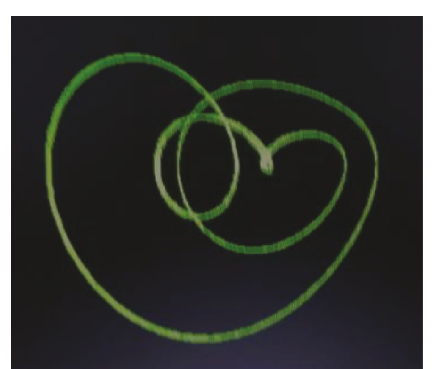

(b)

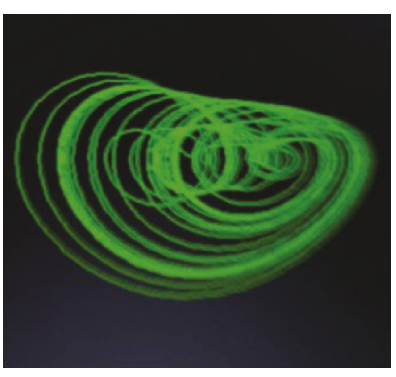

(c)

FIGURE 13: Experimental observation of (a) 2-scroll chaotic behavior; coexistence of (b) period-3 behavior and (c) 1-scroll chaotic behavior on the $x-y$ plane.

We first select the system parameter as $a=0.5, b=0.8$, $c=0.6, d=3, k=1$. When setting $R_{0}=10 \mathrm{k} \Omega$ and $C_{0}=500$ $\mathrm{nF}$, the other resistances are derived as $R_{1}=33.3 k, R_{2}=100$ $k, R_{3}=200 k, R_{4}=50 k, R_{5}=1 k, R_{6}=1.667 k$, and $R_{7}=125 k$. The experimental result of the 2 -scroll chaotic attractor on $x-y$ plane captured from the analog oscilloscope is depicted in Figure 13(a), which agrees well with the numerical simulation in Figure 3.

To experimentally confirm the coexisting attractors, we consider the system parameters as $a=0.5, b=0.8, c=0.6$, $d=3$, and $k=0.95$. In this case, the parameter values of circuit element in Figure 12 are invariable except for $R_{4}=$ $52.63 \mathrm{k}$ and $R_{5}=1.053 \mathrm{k}$. We switch on and off the power supply for randomly selecting the initial states, the experimental results in Figures 13(b) and 13(c) show the period-3 mode and 1-scroll chaotic attractor, which, respectively, agrees well with the numerical simulations in Figure 8 of initial conditions $(0.01,0.23,0.09)$ and $(0.01,0.4,0)$.

\section{Conclusions}

As the fourth basic circuit element, the memristor builds the missing bridges between flux and charge. The memory characteristic of memristor will lead to complicated dynamical behaviors of oscillating systems. The jerk system is of interest within the field of nonlinear dynamics for the simple mathematical form. Therefore, it is worth to study the dynamics of the memristor-based jerk system. In this paper, we propose a 3D jerk system by introducing a generalized memristive device. The dynamical behavior of the system is sensitive to the initial conditions, which results in the coexistence of multiple attractors. And there exist different transition behaviors depending on the selection of the parameters and initial values. Thereby, it is difficult for the third party to reconstruct the accurate state space of the reported system since the dynamics extremely rely on the system parameters, initial values, and time evolution, which are significant for secure communication.

\section{Data Availability}

The figure data and table data used to support the findings of this study are included within the article.

\section{Conflicts of Interest}

The authors declare that there is no conflict of interest regarding the publication of this paper.

\section{Acknowledgments}

This work was supported by the National Natural Science Foundation of China (no. 51577046), the State Key Program of National Natural Science Foundation of China (no. 51637004), the National Key Research and Development Plan "important scientific instruments and equipment development" (no. 2016YFF0102200), Science and Technology Program of Hunan Province (no. 2016TP1021), the Research Foundation of Education Bureau of Hunan Province of China (no. 16B113), and Natural Science Foundation of Hunan Province (No. 2016JJ4036).

\section{References}

[1] L. Chua, "Memristor-the missing circuit element," IEEE Transactions on Circuit Theory, vol. 18, no. 5, pp. 507-519, 1971.

[2] L. O. Chua and S. M. Kang, "Memristive devices and systems," Proceedings of the IEEE, vol. 64, no. 2, pp. 209-223, 1976.

[3] D. B. Strukov, G. S. Snider, D. R. Stewart, and R. S. Williams, "The missing memristor found," Nature, vol. 453, no. 7191, pp. 80-83, 2008.

[4] Y. Zhao, Y. Jiang, J. Feng, and L. Wu, "Modeling of memristorbased chaotic systems using nonlinear Wiener adaptive filters based on backslash operator," Chaos, Solitons \& Fractals, vol. 87, pp. 12-16, 2016.

[5] Y. Zhao, X. Zhang, J. Xu, and Y. Guo, "Identification of chaotic memristor systems based on piecewise adaptive Legendre filters," Chaos, Solitons \& Fractals, vol. 81, pp. 315-319, 2015.

[6] M. Di Ventra, Y. V. Pershin, and L. O. Chua, "Circuit elements with memory: memristors, memcapacitors, and meminductors," Proceedings of the IEEE, vol. 97, no. 10, pp. 1717-1724, 2009.

[7] K. Rajagopal, A. Akgul, S. Jafari, and B. Aricioglu, "A chaotic memcapacitor oscillator with two unstable equilibriums and its fractional form with engineering applications," Nonlinear Dynamics, vol. 91, no. 2, pp. 957-974, 2018.

[8] V. T. Pham, S. Jafari, S. Vaidyanathan, C. Volos, and X. Wang, "A novel memristive neural network with hidden attractors 
and its circuitry implementation," Science China Technological Sciences, vol. 59, no. 3, pp. 358-363, 2016.

[9] Y. B. Zhao, C. K. Tse, J. C. Feng, and Y. C. Guo, “Application of memristor-based controller for loop filter design in chargepump phase-locked loops," Circuits, Systems, and Signal Processing, vol. 32, no. 3, pp. 1013-1023, 2013.

[10] C. Yakopcic, T. M. Taha, G. Subramanyam, R. E. Pino, and S. Rogers, "A memristor device model," IEEE Electron Device Letters, vol. 32, no. 10, pp. 1436-1438, 2011.

[11] A. Buscarino, L. Fortuna, M. Frasca, L. V. Gambuzza, and G. Sciuto, "Memristive chaotic circuits based on cellular nonlinear networks," International Journal of Bifurcation and Chaos, vol. 22, no. 3, article 1250070, 2012.

[12] H. Bao, N. Wang, B. Bao, M. Chen, P. Jin, and G. Wang, "Initial condition-dependent dynamics and transient period in memristor-based hypogenetic jerk system with four line equilibria," Communications in Nonlinear Science and Numerical Simulation, vol. 57, pp. 264-275, 2018.

[13] B. Bao, T. Jiang, G. Wang, P. Jin, H. Bao, and M. Chen, “Twomemristor-based Chua's hyperchaotic circuit with plane equilibrium and its extreme multistability," Nonlinear Dynamics, vol. 89, no. 2, pp. 1157-1171, 2017.

[14] D. Kim, M. Jin, and P. H. Chang, "Control and synchronization of the generalized Lorenz system with mismatched uncertainties using backstepping technique and time-delay estimation," International Journal of Circuit Theory and Applications, vol. 45, no. 11, pp. 1833-1848, 2017.

[15] Z. Wang, W. Sun, Z. Wei, and S. Zhang, "Dynamics and delayed feedback control for a 3D jerk system with hidden attractor," Nonlinear Dynamics, vol. 82, no. 1-2, pp. 577-588, 2015.

[16] H. Saberi Nik, S. Effati, and J. Saberi-Nadjafi, "New ultimate bound sets and exponential finite-time synchronization for the complex Lorenz system," Journal of Complexity, vol. 31, no. 5, pp. 715-730, 2015.

[17] S. Jafari, A. Ahmadi, A. J. M. Khalaf, H. R. Abdolmohammadi, V. T. Pham, and F. E. Alsaadi, "A new hidden chaotic attractor with extreme multi-stability," AEU - International Journal of Electronics and Communications, vol. 89, pp. 131-135, 2018.

[18] Y.-X. Tang, A. Jalil M Khalaf, K. Rajagopal, V.-T. Pham, S. Jafari, and Y. Tian, "A new nonlinear oscillator with infinite number of coexisting hidden and self-excited attractors," Chinese Physics B, vol. 27, no. 4, article 040502, 2018.

[19] V. T. Pham, S. Vaidyanathan, C. K. Volos, S. Jafari, and $\mathrm{X}$. Wang, "A chaotic hyperjerk system based on memristive device," in Advances and Applications in Chaotic Systems, Springer, Cham, 2016.

[20] B. Bao, X. Zou, Z. Liu, and F. Hu, "Generalized memory element and chaotic memory system," International Journal of Bifurcation and Chaos, vol. 23, no. 8, article 1350135, 2013.

[21] Z. Galias, "Automatized search for complex symbolic dynamics with applications in the analysis of a simple memristor circuit," International Journal of Bifurcation and Chaos, vol. 24, no. 7, article 1450104, 2014.

[22] Y. V. Pershin and M. Di Ventra, "Memory effects in complex materials and nanoscale systems," Advances in Physics, vol. 60 , no. 2 , pp. 145-227, 2011.

[23] D. Batas and H. Fiedler, "A memristor SPICE implementation and a new approach for magnetic flux-controlled memristor modeling," IEEE Transactions on Nanotechnology, vol. 10, no. 2, pp. 250-255, 2011.
[24] V. T. Pham, S. Vaidyanathan, C. K. Volos, S. Jafari, N. V. Kuznetsov, and T. M. Hoang, "A novel memristive time-delay chaotic system without equilibrium points," The European Physical Journal Special Topics, vol. 225, no. 1, pp. 127-136, 2016.

[25] J. C. Sprott, "Some simple chaotic jerk functions," American Journal of Physics, vol. 65, no. 6, pp. 537-543, 1997.

[26] J. Kengne, Z. T. Njitacke, and H. B. Fotsin, "Dynamical analysis of a simple autonomous jerk system with multiple attractors," Nonlinear Dynamics, vol. 83, no. 1-2, pp. 751-765, 2016.

[27] J. Kengne, A. N. Negou, and D. Tchiotsop, “Antimonotonicity, chaos and multiple attractors in a novel autonomous memristor-based jerk circuit," Nonlinear Dynamics, vol. 88, no. 4, pp. 2589-2608, 2017.

[28] V. T. Pham, S. Jafari, X. Wang, and J. Ma, "A chaotic system with different shapes of equilibria," International Journal of Bifurcation and Chaos, vol. 26, no. 4, article 1650069, 2016.

[29] V. T. Pham, C. Volos, S. Jafari, and T. Kapitaniak, "A novel cubic-equilibrium chaotic system with coexisting hidden attractors: analysis, and circuit implementation," Journal of Circuits, Systems and Computers, vol. 27, no. 4, article 1850066, 2018.

[30] J. He, S. Yu, and J. Lü, "Constructing higher-dimensional nondegenerate hyperchaotic systems with multiple controllers," International Journal of Bifurcation and Chaos, vol. 27, no. 9, article 1750146, 2017.

[31] C. L. Li, H. M. Li, W. Li et al., "Dynamics, implementation and stability of a chaotic system with coexistence of hyperbolic and non-hyperbolic equilibria," AEU - International Journal of Electronics and Communications, vol. 84, pp. 199-205, 2018.

[32] C. H. Wang, H. Xu, and F. Yu, “A novel approach for constructing high-order Chua's circuit with multi-directional multi-scroll chaotic attractors," International Journal of Bifurcation and Chaos, vol. 23, no. 2, article 1350022, 2013.

[33] Q. Wang, S. Yu, C. Guyeux, J. M. Bahi, and X. Fang, "Theoretical design and circuit implementation of integer domain chaotic systems," International Journal of Bifurcation and Chaos, vol. 24, no. 10, article 1450128, 2014.

[34] A. T. Azar, C. Volos, N. A. Gerodimos et al., "A novel chaotic system without equilibrium: dynamics, synchronization, and circuit realization," Complexity, vol. 2017, Article ID 7871467, 11 pages, 2017. 


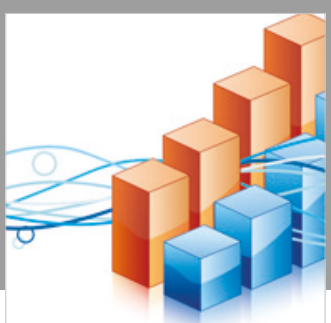

Advances in

Operations Research

\section{-n-m}
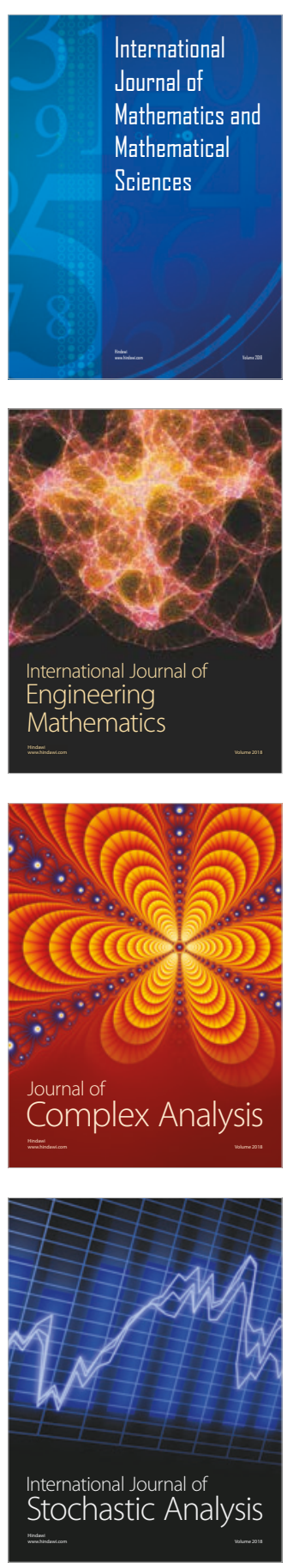
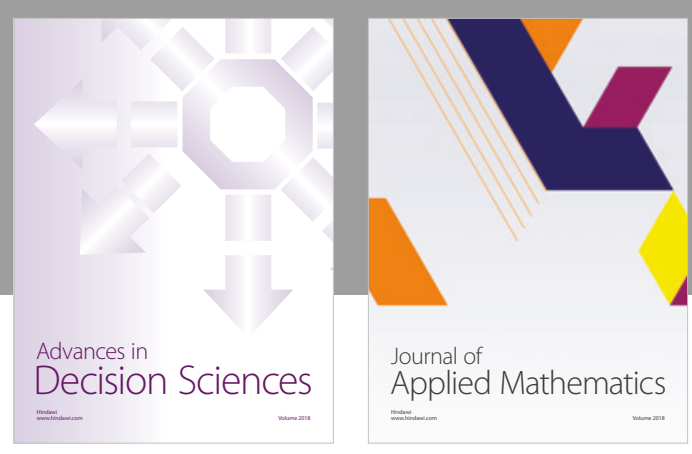

Journal of

Applied Mathematics
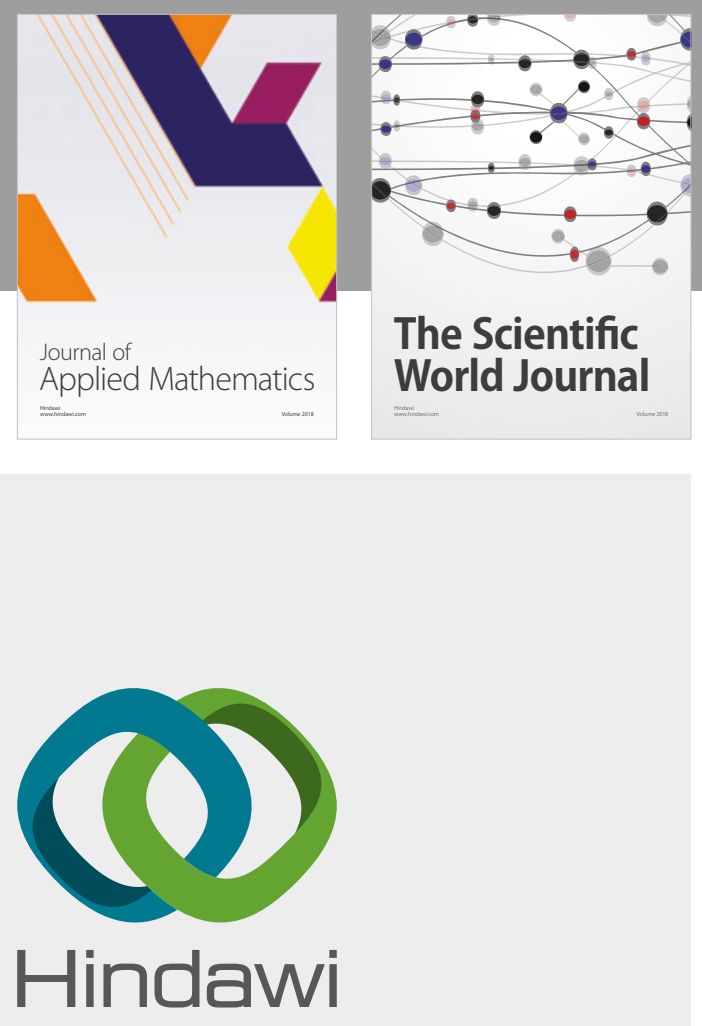

Submit your manuscripts at

www.hindawi.com

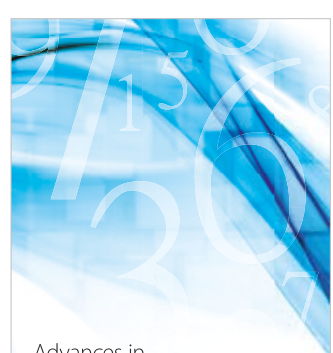

Advances in
Numerical Analysis
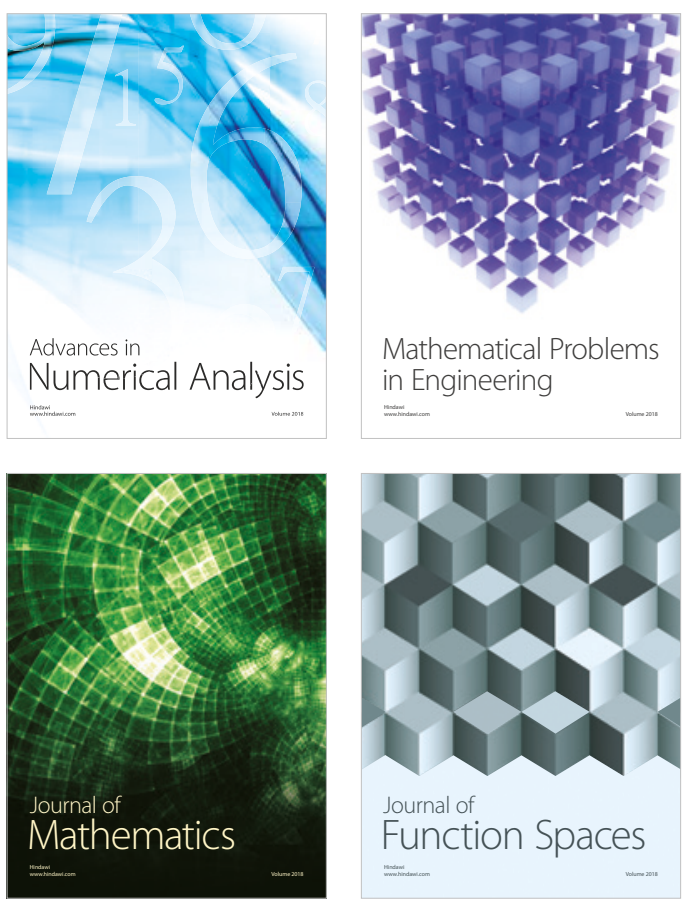

Mathematical Problems in Engineering

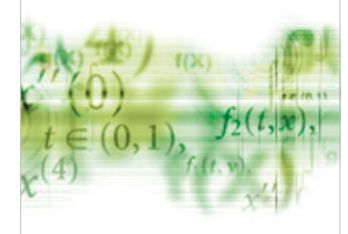

International Journal of

Differential Equations

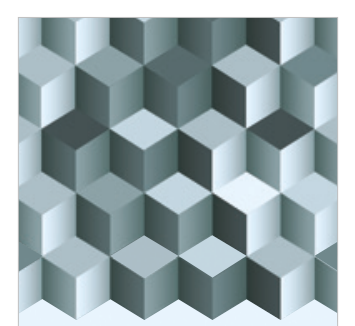

Journal of

Function Spaces
The Scientific

World Journal

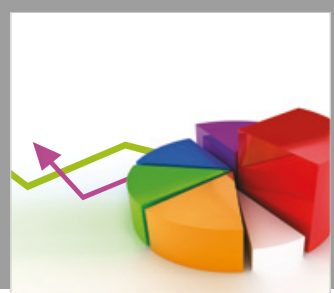

Journal of

Probability and Statistics
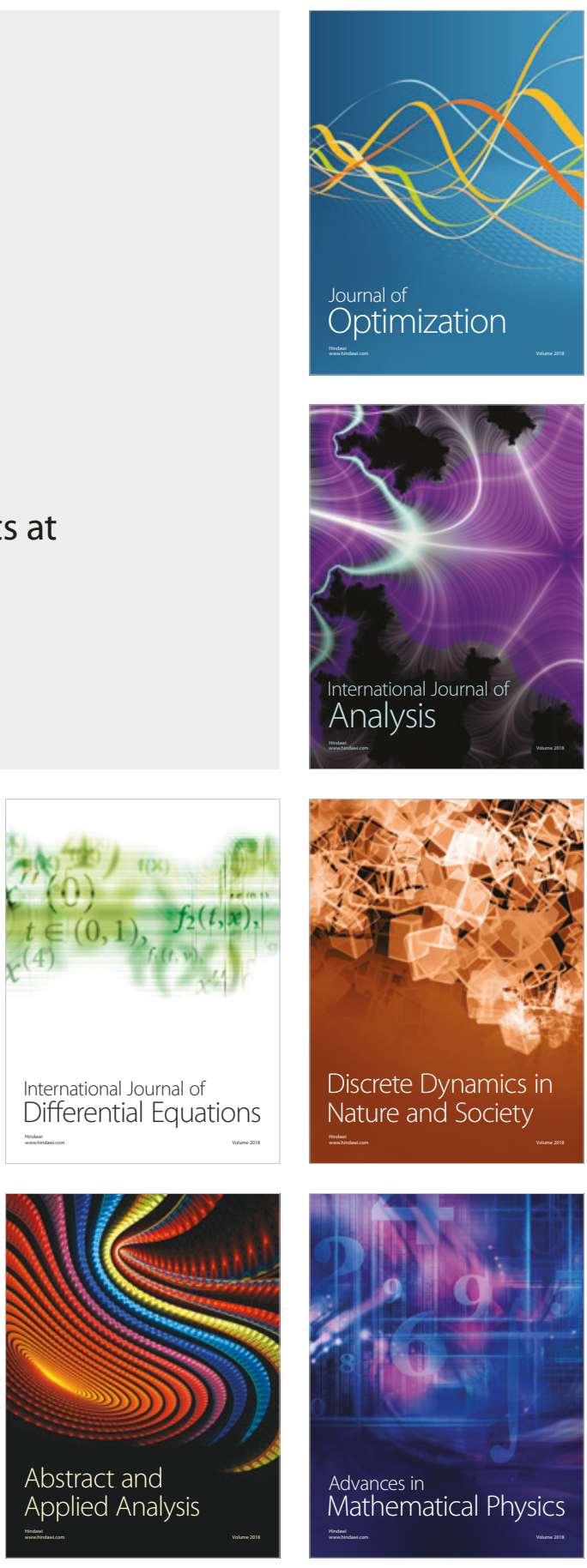